\title{
Decay Mode Solutions to $(2+1)$-Dimensional Burgers Equation, Cylindrical Burgers Equation and Spherical Burgers Equation
}

\author{
Xiangzheng Li' ${ }^{1}$, Jinliang Zhang1, Mingliang Wang1,2 \\ ${ }^{1}$ School of Mathematics \& Statistcs, Henan University of Science \& Technology, Luoyang, China \\ ${ }^{2}$ School of Mathematics \& Statistcs, Lanzhou University, Lanzhou, China \\ Email: xiangzheng_li@qq.com
}

How to cite this paper: Li, X.Z., Zhang, J.L. and Wang, M.L. (2017) Decay Mode Solutions to $(2+1)$-Dimensional Burgers Equation, Cylindrical Burgers Equation and Spherical Burgers Equation. Journal of Applied Mathematics and Physics, 5, 10091015.

https://doi.org/10.4236/jamp.2017.55088

Received: April 13, 2017

Accepted: May 9, 2017

Published: May 15, 2017

Copyright $\odot 2017$ by authors and Scientific Research Publishing Inc. This work is licensed under the Creative Commons Attribution International License (CC BY 4.0).

http://creativecommons.org/licenses/by/4.0/ c) (i) Open Access

\begin{abstract}
Three $(2+1)$-dimensional equations-Burgers equation, cylindrical Burgers equation and spherical Burgers equation, have been reduced to the classical Burgers equation by different transformation of variables respectively. The decay mode solutions of the Burgers equation have been obtained by using the extended $\left(\frac{G^{\prime}}{G}\right)$-expansion method, substituting the solutions obtained into the corresponding transformation of variables, the decay mode solutions of
\end{abstract} the three $(2+1)$-dimensional equations have been obtained successfully.

\section{Keywords}

Decay mode Solution, $(2+1)$-Burgers Equation, $(2+1)$-Cylindrical Burgers Equation, $(2+1)$-Spherical Burgers Equation,

Transformation of Variables, Extended $\left(\frac{G^{\prime}}{G}\right)$-Expansion Method

\section{Introduction}

Many famous nonlinear evolution equations such as Korteweg-de Vries (KdV), Modified KdV (mKdV), Kadomstev-Perviashvili (KP), Coupled KP and Zakharov-Kuznetsov (ZK) have been obtained by using the standard reductive perturbation method in nonlinear propagation of dust-acoustic wave, especially, the dust-acoustic solitary wave (DASW) in space and laboratory plasma [1]-[7]. Recent theoretical studies for ion-acoustic/dust-acoustic waves show that the properties of solitary waves in bounded nonplanar cylindrical/spherical geometry differ from that in unbounded planar geometry. The effects of dissipation on the propagation of soliton waves are scarcely discussed, especially, for nonplanar 
waves [8]. A dissipative cylindrical/spherical $\mathrm{KdV}$ is obtained by using the standard reductive perturbation method in Ref. [8]. It is known that the transverse perturbations always exist in the higher-dimensional system. Anisotropy is introduced into the system and the wave structure and stability are modified by the transverse perturbation. A spherical KP (SKP) equation is obtained by using the standard reductive perturbation method [9]. The cylindrical KP Equation (CKP) is also be introduced by Johnson [10] [11] to describe surface wave in a shallow incompressible fluid.

Following the extension sense of the KP equation Ref. [12] developed the $(2+$ 1)-Burgers equation in the form

$$
\left(u_{t}+u u_{x}+v u_{x x}\right)_{x}+\lambda u_{y y}=0,
$$

where $v$ is a constant that defines the kinematic viscosity, $\lambda$ is a constant. If surface tension is weak compared to gravitational forces, then $\lambda>0$ is used. However if surface tension is strong, then $\lambda<0$ is used. The kink solutions and periodic solutions were obtained by using the tanh-coth method, $N$-soliton solutions were established by applying the powerful Hirota's bilinear method in Ref. [12].

In this work, following the extension sense of $(2+1)$-Burgers equation [12] and cylindrical KP equation [10] [11] [13] [14] the $(2+1)$-cylindrical Burgers equation can be developed in the form

$$
\left(u_{t}+u u_{x}+v u_{x x}+\frac{1}{2 t} u\right)_{x}+\frac{\lambda}{t^{2}} u_{y y}=0,
$$

where $v$ is a constant that defines the kinematic viscosity, $\lambda$ is a constant.

Following the $(2+1)$-Burgers equation and spherical KP equation [9], the (2 $+1)$-spherical Burgers equation can be developed in the form

$$
\left(u_{t}+u u_{x}+v u_{x x}+\frac{1}{t} u\right)_{x}+\frac{\lambda}{t^{2}}\left(u_{y y}+\frac{1}{y} u_{y}\right)=0 \text {, }
$$

where $v$ is a constant that defines the kinematic viscosity, $\lambda$ is a constant.

The nonlinear evolution equation can describe various motions. So it is important to study their exact solutions. There exist many kinds of solutions to some integrable equations such as soliton, complexiton, negaton, rational and periodic solutions [13]. Besides these solutions, there exists the decay mode soliton which was proposed by Nakamura [13] [15]. Physically, the only difference between soliton and decay mode is that soliton is absolutely stable while decay mode vanishes eventually as time passes. The decay mode solutions for the cylindrical KP equation were obtained by the Backlund transformation and Hirota method in Ref. [13]. The solutions of the CKP in Ref. [13] were expressed in terms of the Airy functions. The 1-decay mode and 2-decay mode solutions of the CKP equation have been obtained in terms of the nonlinear transformation derived by using the simplified homogeneous balance method (SHB) [14] [16] [17] in Ref. [14].

In the present paper, the aim is to study the decay mode solutions of Equations (1), (2) and (3). The paper is organized as follows: In Section 2, making 
transformation of variables, by which reduction of $(2+1)$-dimensional Burgers equation, cylindrical Burgers equation and spherical Burgers equation to the classical Burgers equation; In Section 3, the decay mode solutions of the classical Burgers equation are obtained by using the extended $\left(\frac{G^{\prime}}{G}\right)$-expansion method (the original $\left(\frac{G^{\prime}}{G}\right)$-expansion method can be found in Ref. [18]); In Section 4, using the results obtained in Section 3, the decay mode solutions of Equations (1), (2) and (3) can be obtained by using different transformations of variables, respectively; In Section 5, some conclusions are made.

\section{Reduction of $(2+1)$-Dimensional Burgers Equation, Cylindrical Burgers Equation and Spherical Burgers Equation}

In Equation (1), assume that

$$
u=w(\xi, t), \quad \xi=x-q(y, t),
$$

where $q=q(y, t)$ is to be determined later. Substituting Equation (4) into Equation (1), yields an equation as follows

$$
\frac{\partial}{\partial \xi}\left(w_{t}+w w_{\xi}+v w_{\xi \xi}\right)-\lambda q_{y y} w_{\xi}+\left(\lambda q_{y}^{2}-q_{t}\right) w_{\xi \xi}=0 .
$$

Setting the coefficients of $w_{\xi}$ and $w_{\xi \xi}$ to zero, yields

$$
-\lambda q_{y y}=0, \lambda q_{y}^{2}-q_{t}=0
$$

the system (6) admits a solution:

$$
q(y, t)=\mu y+\lambda \mu^{2} t,
$$

where $\mu$ is a nonzero arbitrary constant. Using Equation (7) the expression (4) becomes

$$
u=w(\xi, t), \quad \xi=x-\left(\mu y+\lambda \mu^{2} t\right),
$$

and after integrating Equation (5) with respect to $\xi$ once and taking the constant of integration to zero, Equation (5) becomes the classical Burgers equation for $w=w(\xi, t)$

$$
w_{t}+w w_{\xi}+v w_{\xi \xi}=0 .
$$

From the discussion above, the conclusion can be made that the $(2+1)$-dimensional Burgers Equation (1) for $u=u(x, y, t)$ is reduced to the Burgers Equation (9) for $w=w(\xi, t)$ by using the transformation of variables (8), if $w(\xi, t)$ is a solution of Burgers Equation (9), substituting it into Equation (8), then the exact solution of the $(2+1)$-Burgers equation can be obtained.

Similarly, the $(2+1)$-dimensional cylindrical Burgers Equation (2) for $u=u(x, y, t)$ is reduced to Equation (9) for $w=w(\xi, t)$ by using the transformation of variables

$$
u=w(\xi, t), \quad \xi=x-\left(\frac{1}{4 \lambda} y^{2} t+\mu y t+\lambda \mu^{2} t\right),
$$


where $\mu$ is a nonzero arbitrary constant, if $w(\xi, t)$ is a solution of Equation (9), and substituting it into Equation (10), the exact solution of Equation (2) can be obtained.

The conclusion can be made that the $(2+1)$-dimensional spherical Burgers Equation (3) for $u=u(x, y, t)$ is reduced to Equation (9) for $w=w(\xi, t)$ by using the transformation of variables

$$
u=w(\xi, t), \quad \xi=x-\left(\frac{1}{4 \lambda} y^{2} t+\mu\right),
$$

where $\mu$ is a nonzero arbitrary constant, if $w(\xi, t)$ is a solution of Equation (9), and substituting it into Equation (11), the exact solution of Equation (3) can be obtained.

\section{Decay Mode Solution of Burgers Equation}

Considering the homogeneous balance between $w w_{\xi}$ and $w_{\xi \xi}$ in Equation (9) $(2 m+1=m+2 \rightarrow m=1)$, according to the extended $\left(\frac{G^{\prime}}{G}\right)$-expansion method, a suppose can be made that the solution of Equation (9) is of the form

$$
w=v_{1}(\xi, t) \frac{G^{\prime}(\varphi)}{G(\varphi)}+v_{0}(\xi, t),
$$

where $v_{1}=v_{1}(\xi, t), \varphi=\varphi(\xi, t), v_{0}=v_{0}(\xi, t)$ are to be determined later (Noticed that in the original $\left(\frac{G^{\prime}}{G}\right)$-expansion, $v_{1}, v_{0}$ are constants, $\varphi=\xi=x-v t$ is traveling waves), and $G=G(\varphi)$ satisfies the second order LODE

$$
G^{\prime \prime}+\delta G=0
$$

where $\delta$ is a constant to be determined later.

When $\delta<0$, ODE (13) has general solution

$$
G(\varphi)=A \cosh (\sqrt{-\delta} \varphi)+B \sinh (\sqrt{-\delta} \varphi), \quad A, B \text { are constants }
$$

then

$$
\frac{G^{\prime}(\varphi)}{G(\varphi)}=\sqrt{-\delta} \frac{A \sinh (\sqrt{-\delta} \varphi)+B \cosh (\sqrt{-\delta} \varphi)}{A \cosh (\sqrt{-\delta} \varphi)+B \sinh (\sqrt{-\delta} \varphi)}
$$

Choose $B=0$, then

$$
\frac{G^{\prime}(\varphi)}{G(\varphi)}=\sqrt{-\delta} \tanh (\sqrt{-\delta} \varphi)
$$

Now our main goal is to determine $v_{1}=v_{1}(\xi, t), \varphi=\varphi(\xi, t), v_{0}=v_{0}(\xi, t)$ and constant $\delta$, such that expression (12) satisfies Equation (9).

Substituting Equation (12) into the left side of Equation (9), collecting the coefficients of each power of $\left(\frac{G^{\prime}}{G}\right)^{i},(i=0,1,2,3)$, setting each coefficient to zero, the PDEs can be obtained for $v_{1}=v_{1}(\xi, t), \varphi=\varphi(\xi, t), v_{0}=v_{0}(\xi, t)$ as 
follows

$$
\begin{gathered}
\left(\frac{G^{\prime}}{G}\right)^{3}:-v_{1} \varphi_{\xi}\left(v_{1}-2 v \varphi_{\xi}\right)=0 \\
\left(\frac{G^{\prime}}{G}\right)^{2}:-2 v \varphi_{\xi} v_{1 \xi}-v_{1}\left(\varphi_{t}+v_{0} \varphi_{\xi}-v_{1 \xi}+v \varphi_{\xi \xi}\right)=0 \\
\left(\frac{G^{\prime}}{G}\right): v_{1 t}-\delta v_{1}^{2} \varphi_{\xi}+v_{1}\left(2 v \delta \varphi_{\xi}^{2}+v_{0 \xi}\right)+v_{0} v_{1 \xi}+v v_{1 \xi \xi}=0, \\
\left(\frac{G^{\prime}}{G}\right)^{0}: v_{0 t}+v_{0} v_{0 \xi}-2 v \delta \varphi_{\xi} v_{1 \xi}-\delta v_{1}\left(\varphi_{t}+v_{0} \varphi_{\xi}+v \varphi_{\xi \xi}\right)+v v_{0 \xi \xi}=0
\end{gathered}
$$

Simplifying Equations (16)-(19), then

$$
\begin{gathered}
v_{1}=2 v \varphi_{\xi}, \\
-2 v \varphi_{\xi}\left(\varphi_{t}+v_{0} \varphi_{\xi}+v \varphi_{\xi \xi}\right)=0, \\
2 v \frac{\partial}{\partial \xi}\left(\varphi_{t}+v_{0} \varphi_{\xi}+v \varphi_{\xi \xi}\right)=0, \\
\left(v_{0 t}+v_{0} v_{0 \xi}+v v_{0 \xi \xi}\right)-2 \delta v \varphi_{\xi}\left(\varphi_{t}+v_{0} \varphi_{\xi}-3 v \varphi_{\xi \xi}\right)=0 .
\end{gathered}
$$

Noticed that in Equations (20)-(23), if

$$
\varphi=v_{0}=\frac{\xi}{t}, \text { then } v_{1}=2 v \varphi_{\xi}=\frac{2 v}{t},
$$

Equations (20)-(23) are satisfied completely for arbitrary constant $\delta<0$.

Substituting Equation (15) and Equation (24) into Equation (12), the decay mode solution for Equation (9) can be expressed as follows

$$
w(\xi, t)=\frac{2 v \sqrt{-\delta}}{t} \tanh \left(\frac{\sqrt{-\delta} \xi}{t}\right)+\frac{\xi}{t}, \delta<0 .
$$

As far as we know, the solution (25) has never seen in early literatures.

\section{Decay Mode Solutions of $(2+1)$-Burgers Equation, Cylindrical Burgers Equation and Spherical Burgers Equation}

Substituting Equation (25) into Equation (8), the decay mode solution for Equation (1) can be obtained as follows

$$
u(x, y, t)=\frac{2 v \sqrt{-\delta}}{t} \tanh \left(\frac{\sqrt{-\delta} \xi}{t}\right)+\frac{\xi}{t},
$$

where $\delta<0$ and $\xi=x-\left(\mu y+\lambda \mu^{2} t\right)$.

Substituting Equation (25) into Equation (10), the decay mode solution for Equation (2) can be obtained as follows

$$
u(x, y, t)=\frac{2 v \sqrt{-\delta}}{t} \tanh \left(\frac{\sqrt{-\delta} \xi}{t}\right)+\frac{\xi}{t},
$$

where $\delta<0$ and $\xi=x-\left(\frac{1}{4 \lambda} y^{2} t+\mu y t+\lambda \mu^{2} t\right)$. 
Substituting Equation (25) into Equation (11), the decay mode solution for Equation (3) can be obtained as follows

$$
u(x, y, t)=\frac{2 v \sqrt{-\delta}}{t} \tanh \left(\frac{\sqrt{-\delta} \xi}{t}\right)+\frac{\xi}{t},
$$

where $\delta<0$ and $\xi=x-\left(\frac{1}{4 \lambda} y^{2} t+\mu\right)$.

\section{Conclusion}

In this paper, by making corresponding transformation of variables, the $(2+1)$ dimensional Burgers equation, $(2+1)$-dimensional cylindrical Burgers equation and $(2+1)$-dimensional spherical Burgers equation are all reduced to the classical Burgers equation, which can be solved by using extended $\left(\frac{G^{\prime}}{G}\right)$-expansion method to obtain a novel type of decay mode solution. Substituting the novel solution of the Burgers equation into the corresponding transformation of variables, the decay mode solutions of the $(2+1)$-dimensional Burgers equation, $(2+1)$-dimensional cylindrical Burgers equation and $(2+1)$-dimensional spherical Burgers equation have been obtained for the first time, respectively. The analysis may be extended to other works to make further progress.

\section{Acknowledgements}

The authors would like to thank the Editorand anonymous referees for their helpful suggestions and valuable comments. The project supported in part by the National Natural Science Foundation of China (Grant No. 11301153, 11601225) and The Doctoral Foundation of Henan University of Science and Technology (Grant No. 09001562) and The Science and Technology Innovation Platform of Henan University of Science and Technology(Grant No.2015XPT001).

\section{References}

[1] Wang, H.Y. and Zhang, K.B. (2013) Dust-Acoustic Waves in a Two-Temperature Nonthermal Dusty Plasma. Journal of Sichuan Normal University, 36, 911-914. (In Chinese).

[2] Lü, X., Chen, S.T. and Ma, W.X. (2016) Constructing Lump Solutions to a Generalized Kadomtsev-Petviashvili-Boussinesq Equation. Nonlinear Dynamics, 86, 523 534. https://doi.org/10.1007/s11071-016-2905-Z

[3] Lü, X. and Ma, W.X. (2016) Study of Lump Dynamics Based on a Dimensionally Reduced Hirota Bilinear Equation. Nonlinear Dynamics, 85, 1217-1222. https://doi.org/10.1007/s11071-016-2755-8

[4] Lü, X., Ma, W.X., Zhou, Y. and Khalique, C.M. (2016) Rational Solutions to an Extended Kadomtsev-Petviashvili-Like Equation with Symbolic Computation. Computers \& Mathematics with Applications, 71, 1560-1567.

[5] Lü, X., Ma, W.X., Chen, S.T. and Khalique, C.M. (2016) A Note on Rational Solutions to a Hirota-Satsuma-Like Equation. Applied Mathematics Letters, 58, 13-18.

[6] Lü, X., Ma, W.X., Yu, J. and Khalique, C.M. (2016) Solitary Waves with the Madelung Fluid Description: A Generalized Derivative Nonlinear Schrodinger Equation. 
Communications in Nonlinear Science and Numerical Simulation, 31, 40-46.

[7] Lü, X. and Lin, F.H. (2016) Soliton Excitations and Shape-Changing Collisions in Alphahelical Proteins with Interspine Coupling at Higher Order. Communications in Nonlinear Science and Numerical Simulation, 32, 241-261.

[8] Xue, J.K. (2004) Cylindrical and Spherical Ion-Acoustic Solitary Waves with Dissipative Effect. Physics Letters A, 322, 225-230.

[9] Xue, J.K. (2003) A Spherical KP Equation for Dust Acoustic Waves. Physics Letters $A, 314,479-483$.

[10] Johnson, R.S. (1980) Water Waves and Korteweg-de Vries Equations. Journal of Fluid Mechanics, 97, 701-709. https://doi.org/10.1017/S0022112080002765

[11] Johnson, R.S. (1997) A Modern Introduction to the Mathematical Theory of Water Waves. Cambridge University Press, Cambridge. https://doi.org/10.1017/CBO9780511624056

[12] Wazwaz, A.M. (2008) Multiple-Front Solutions for the Burgers-Kadomtsev-Petviashvili Equation. Applied Mathematics and Computation, 200, 437-443.

[13] Deng, S.F. (2012) The Decay Mode Solutions for Cylindrical KP Equation. Applied Mathematics and Computation, 218, 5974-5981.

[14] Wang, M.L., Zhang, J.L. and Li, X.Z. (2016) Decay Mode Solutions to Cylindrical KP Equation. Applied Mathematics Letters, 62, 29-34.

[15] Nakamura, A. and Hirota, R. (1981) Decay Mode Solution of the Two-Dimensional KdV Equation and the Generalized Bäcklund Transformation. Journal of Physics A, 42, 2456-2462.

[16] Wang, M.L. and Li, X.Z. (2014) Simplified Homogeneous Balance Method and Its Application to the Whitham-Broer-Kaup Model Equations. Journal of Applied Mathematics and Physics, 2, 823-827. https://doi.org/10.4236/jamp.2014.28091

[17] Wang, M.L., Zhang, J.L. and Li, X.Z. (2017) N-Dimensional Auto-Backlund Transformation and Exact Solutions to n-Dimensional Burgers System. Applied Mathematics Letters, 63, 46-52.

[18] Wang, M.L., Li, X.Z. and Zhang, J.L. (2008) The (GG)-Expansion Method and Travelling Wave Solutions of Nonlinear Evolution Equations in Mathematical Physics. Physics Letters A, 372, 417-423.

\section{Submit or recommend next manuscript to SCIRP and we will provide best service for you:}

Accepting pre-submission inquiries through Email, Facebook, LinkedIn, Twitter, etc. A wide selection of journals (inclusive of 9 subjects, more than 200 journals)

Providing 24-hour high-quality service

User-friendly online submission system

Fair and swift peer-review system

Efficient typesetting and proofreading procedure

Display of the result of downloads and visits, as well as the number of cited articles

Maximum dissemination of your research work

Submit your manuscript at: http://papersubmission.scirp.org/

Or contact jamp@scirp.org 